\title{
Serum Tissue Non Specific Alkaline Phosphatase Isoenzyme Level and Severity of Chronic Periodontitis
}

\author{
Gopu Chandran Lenin Perumal ${ }^{*}$ R. Mythili², Senthil Kumar², Subashini Suyambukesan ${ }^{3}$ \\ 'Department of Periodontology, Penang International Dental College, Penang, \\ Malaysia; drgopuchandran@gmail.com \\ ${ }^{2}$ Rajah Muthiah Dental College \& Hospital, Annamalai University, Chidambaram, Tamilnadu, India \\ ${ }^{3}$ Department of Oral medicine \& Radiology, Penang International Dental College, Penang, Malaysia
}

\begin{abstract}
The purpose of this study was to estimate and compare the levels of tissue non-specific alkaline phosphatase isoenzyme in serum of healthy individual and in chronic periodontitis patients with varying severity. Serum samples were obtained from 31individuals. 10 from healthy individuals and 21 from chronic periodontitis patients categorized as mild, moderate and severe based on CAL values. The samples were used to determine the Tissue Non - Specific Alkaline Phosphatase Isoenzyme (TNSALP) level. Evaluation and comparison of the tissue non - specific alkaline phosphatase isoenzyme activity between the control and chronic periodontitis patient group showed a decrease in bone type of tissue non - specific alkaline phosphatase level among mild, moderate and severe chronic periodontitis patients group. The steady decrease in Bone type of TNSALP might help to provide useful information if monitored successively over a period of time.
\end{abstract}

Keywords: Alkaline Phosphatase Isoenzyme, Chronic Periodontitis, Severity of Chronic Periodontitis, Serum Enzymatic Assay, Tissue Non-Specific Alkaline Phosphatase Isoenzyme, TNSALP.

\section{Introduction}

Chronic periodontitis is a longstanding inflammatory disease affecting the supporting structures of the dentition namely the gingiva, periodontal ligament, cementum and adjacent surrounding alveolar bone. Most routine methods to diagnose chronic periodontitis is by clinical evaluation of affected site and measuring the attachment loss followed by radiographic confirmation by identifying bone loss. These methods do not shed light on the status of progression or regression occurring in the diseased sites and also fail to identify newly developing disease sites $^{1,2}$.

For better evaluation of prognosis and effective treatment, it is important to identify the current status of the disease. Changes at both cellular levels and sub - cellular levels show us the current status of the disease and are evaluated or identified using biochemical assay. The most common and widely relied enzyme assay for bone disease is Alkaline Phosphatase (ALP) measurement. Alkaline phosphatase has been found in various periodontal tissues ${ }^{3,4}$ with increased activity noticed during progression of periodontitis ${ }^{5,6}$. Serum level of ALP enzyme can be used as an indicator for bone formation? ${ }^{7}$.

Alkaline phosphatase enzyme consists of a group of true isoenzymes, all glycoproteins, encoded for by at least four different gene loci: tissue-nonspecific, intestinal, placental, and germ-cell ALP. In human serum, tissue-nonspecific (liver, bone, and kidney), intestinal, and placental ALP isoforms contribute to total ALP activity, making clinical interpretation difficult without fractionation of these ALP isoforms. Various methods of differentiating the B-ALP from liver ALP include conventional agarose gel electrophoresis ${ }^{8,9}$, heat and chemical ${ }^{10}$, wheat germ agglutinin precipitation ${ }^{11}$, and wheat germ agglutinin-high performance liquid chromatography. 
Bone form of Tissue Non-Specific Alkaline Phosphatase (B-TNSALP) has been considered to be a good marker in serum for bone formation ${ }^{12}$. Measurement of B-TNSALP in the serum provides a more specific assessment of the metabolic status of bone in normal and pathological conditions. Hence the main purpose of this study was to evaluate and compare the tissue nonspecific isoenzyme of alkaline phosphatase enzyme among healthy individuals and patients with varying severity of chronic periodontitis.

\section{Materials and Methods}

This study was done in division of Periodontics, RMDC \& $\mathrm{H}$, Annamalai University after ethical clearance. Informed consent were obtained from 31 participants who were selected randomly among the patients who visited the division of Periodontics. 10 individuals in control group and 21 patients in test groups of age 30-55 years were selected with the criteria's listed below and are grouped as

Group A: Control group - healthy individuals ( CAL of $0 \mathrm{~mm}$ ) and

Group B: Test groups - Chronic periodontitis patients

$\mathrm{B}_{1}$ : Mild (CAL $\left.1-2 \mathrm{~mm}\right)$;

$\mathrm{B}_{2}$ : Moderate (CAL 3- 4mm) and

$\mathrm{B}_{3}$ : Severe $(\mathrm{CAL} \geq 5 \mathrm{~mm})$

\subsection{Selection Criteria}

Individuals included in this study are selected based on the following criteria. Smokers, malnourished, anemic, subjects taking medicines known to affect periodontal conditions or gingival secretion, any cardiac diseases, hepato-biliary diseases, diabetes, thyroid and parathyroid abnormalities, Viral, fungal or bacterial infection, history of recent trauma or tooth extractions, pregnant or lactating women, women on oral contraceptives, history of systemic antibiotic therapy within 6 months were excluded from the study.

\subsection{Clinical Examinations}

Brief and precise medical and dental history were recorded after informed consent followed by clinical examination. The clinical indices and parameters like Probing Pocket Depth level (PPD) and Clinical Attachment Loss (CAL) were recorded.

\subsection{Sample and Evaluation}

$5 \mathrm{ml}$ of blood samples were collected from all participating individuals after overnight fasting and was allowed to clot in a test tube placed slantingly. After an hour the supernatant serum was extracted and sent to biochemical laboratory for assay. Total alkaline phosphatase enzyme were evaluated and then Tissue Non-Specific Isoenzyme namely Bone (B-TNSALP) and Liver ( $\mathrm{L}_{1}$ and $\mathrm{L}_{2}$ TNSALP) isoenzymes were separated by electrophoresis on agarose gel and visualized and quantified using the Hydragel ISO-PAL K20 kit (Sebia, France), following the manufacturer's recommendations. The values obtained were tabulated in $\%$ for isoenzyme fractions.

\subsection{Data Analysis}

Results were tabulated; Sample's mean and standard deviation for the results were determined. Between each study groups, the results were compared using ANalysis of VAriance (ANOVA) test for the determination of statistical significance. All statistical analysis was performed using standard statistical software. $\mathrm{P}<0.05$ was considered as statistically significant.

\section{Results}

Table 1 and Figure 1 represents the mean value of Bone type Tissue Non-Specific Alkaline Phosphatse Isoenzyme (B - TNSALP). 10 individuals in the control group with a mean ALP level of $44.2 \pm 1.378 .7$ patients in each subgroups of test group were screened for the Bone type of tissue non-specific alkaline phosphatase level. Sub group $\mathrm{B}_{1}$ identified as mild periodontitis patient has an estimated mean value of $39.357 \pm 0.783$. The sub group $B_{2}$, designated as moderate periodontitis group has mean of 33.914 \pm 0.867 . The subgroup $B_{3}$ categorized as severe periodontitis patients has a mean of $24.329 \pm 0.547$ bone type of tissue non-specific alkaline phosphatase isoenzyme in serum.

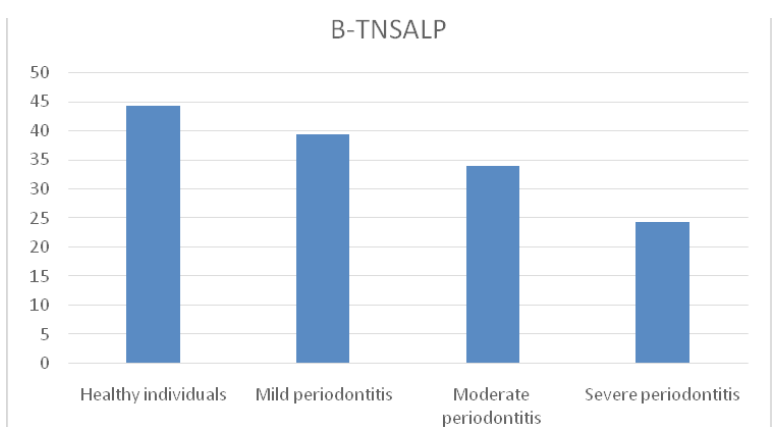

Figure 1. Bone type of Tissue Non Specific Alkaline Phosphatase isoenzyme (B-TNSALP). 
Table 1. Bone type of Tissue Non Specific Alkaline Phosphatase isoenzyme (B-TNSALP)

\begin{tabular}{|c|c|c|c|c|c|c|}
\hline & Group & $\mathrm{N}$ & Mean & Std. Deviation & F-value & P-value \\
\hline \multirow{4}{*}{ Bone (\%) } & Health individuals & 10 & 44.200 & 1.378 & \multirow{4}{*}{576.2} & \multirow{4}{*}{$0.001(\mathrm{~S})$} \\
\hline & Mild periodontitics & 7 & 39.357 & .783 & & \\
\hline & Moderate periodontitics & 7 & 33.914 & .867 & & \\
\hline & Severe periodontitics & 7 & 24.329 & .547 & & \\
\hline
\end{tabular}

Table 2 and Figure 2 represents the mean value of Liver type Tissue Non-Specific Alkaline Phosphatase isoenzyme $\left(\mathrm{L}_{1}\right.$ - TNSALP). 10 individuals in the control group with a mean ALP level of $49.52 \pm 1.339 .7$ patients in each subgroups of test group were screened for the liver type of tissue non-specific alkaline phosphatase level. Sub group $\mathrm{B}_{1}$ identified as mild periodontitis patient has an estimated mean value of $52.557 \pm 0.757$. The sub group $\mathrm{B}_{2}$ designated as moderate periodontitis group has mean of $43.857 \pm 0.624$. The subgroup $B_{3}$ categorized as severe

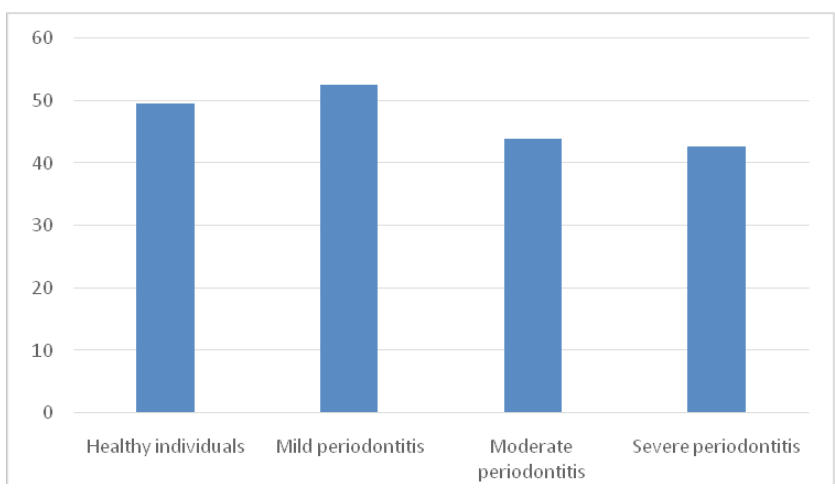

Figure 2. Liver type of tissue nonspecific alkaline phosphatase isoenzyme ( $\mathrm{L}_{1}$-TNSALP). periodontitis patients has a mean of $42.757 \pm 3.661$ liver type of tissue non-specific alkaline phosphatase isoenzyme in serum.

Table 3 and Figure 3 represents the mean value of Liver type Tissue Non-Specific Alkaline Phosphatase isoenzyme ( $\mathrm{L}_{2}$ - TNSALP). 10 individuals in the control group with a mean ALP level of $6.270 \pm 0.157 .7$ patients in each subgroups of test group were screened for the liver type of tissue non-specific alkaline phosphatase level. Sub group $\mathrm{B}_{1}$ identified as mild periodontitis patient has an estimated

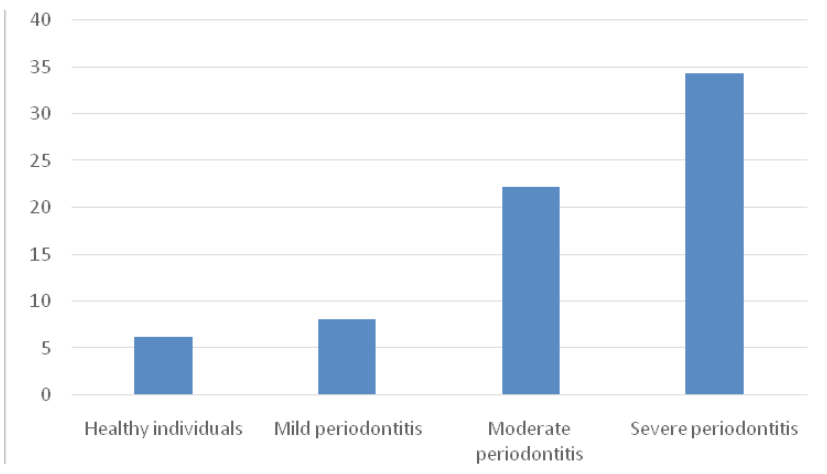

Figure 3. Liver type of Tissue Non Specific Alkaline Phosphatase isoenzyme $\left(\mathrm{L}_{2}\right.$-TNSALP).

Table 3. Liver type of Tissue Non Specific Alkaline Phosphatase isoenzyme ( $\mathrm{L}_{1}$-TNSALP)

\begin{tabular}{llccccc}
\hline \multicolumn{1}{c}{ Group } & N & Mean & Std. Deviation & F-value & P-value \\
\hline \multirow{3}{*}{ Liver 1 (\%) } & Health individuals & 10 & 49.520 & 1.339 & .757 & 41.31 \\
& Mild periodontitics & 7 & 52.557 & .624 & & \\
& Moderate periodontitics & 7 & 43.857 & $3.601(\mathrm{~S})$ \\
& Severe periodontitics & 7 & 42.757 & & \\
\hline
\end{tabular}

Table 2. Liver type of Tissue Non Specific Alkaline Phosphatase isoenzyme ( $\mathrm{L}_{2}$-TNSALP)

\begin{tabular}{llllcr}
\hline \multicolumn{1}{c}{ Group } & N & Mean & Std. Deviation & F-value & P-value \\
\hline \multirow{2}{*}{ Liver 2 (\%) } & Health individuals & 10 & 6.270 & .157 & 18790.6 \\
& Mild periodontitics & 7 & 8.086 & .398 & $.001(\mathrm{~S})$ \\
& Moderate periodontitics & 7 & 22.229 & .304 & .177 \\
& Severe periodontitics & 7 & 34.314 & & \\
\hline
\end{tabular}


mean value of $8.086 \pm 0.398$. The sub group $B_{2}$ designated as moderate periodontitis group has mean of $22.229 \pm$ 0.304 . The subgroup $B_{3}$ categorized as severe periodontitis patients has a mean of $34.314 \pm 0.117$ liver type of tissue non-specific alkaline phosphatase isoenzyme in serum.

\section{Discussion}

The tissue non-specific isoenzyme of alkaline phosphatase was analyzed in both healthy individuals and in persons diagnosed as chronic periodontitis. These subjects diagnosed as chronic periodontitis are further sub grouped based on the clinical attachment loss as mild, moderate and severe.

This study was done using the serum based on two major factor. First, the quantity of sample required to estimate TNSALP is adequate at present only in serum and the second reason is based on the observation made by Requirand ${ }^{13}$. They have evaluated bone type TNSALP in serum and have concluded its role in chronic periodontitis based on the hypothesis that the decrease of bone ALP observed in the PL, a decrease could be expected at the serum levels if some interaction could occur between these two compartments ${ }^{13}$. They have defended that there exists some kind of interaction between two compartments and so that any change in serum of otherwise healthy individuals diagnosed with chronic periodontitis patients, do reflect the same change in the periodontal environment.

In this study, it is noted that there was a steady decrease in the bone type of TNSALP among healthy to mild, moderate and severe patients. Similar results were obtained in the study done by Gibert et al where they have also compared the level of serum bone type of tissue nonspecific alkaline phosphatase isoenzymes between healthy individuals (control) and chronic periodontitis patients ${ }^{14}$. They also inferred that there was a drop in bone type of tissue nonspecific alkaline phosphatase level with severity of diseases. This decrease in bone TNSALP might be attributed to the decreased bone apposition tendency or may also be due to reduced local synthesis or reduced passage into the circulation. Not many studies has been done so far relating ALP isoenzymes with chronic periodontitis. Hence, further studies have to be done with increased sample size and various standardizations and sample sources like saliva and gingival crevicular fluid for confirming the use of TNSALP as a confirmatory assay for disease severity.
In conclusion, the results of this study helps in making use of TNSALP isoenzyme estimation as one of the reliable assay to determine the severity and progression of chronic periodontitis.

\section{References}

1. Page RC. Host response tests for diagnosing periodontal diseases. J Periodontol. 1992; 19:43-8.

2. Fine DH, Mandel ID. Indicators of periodontal disease activity: An evaluation. J Clin Periodontol. 1986; 13:533-46.

3. Cimasoni G. Crevicular Fluid Updated. Basel: Karger; 1983.

4. Nakashima K, Roehrich N, Cimasoni G. Osteocalcin, prostaglandin E2 and alkaline phosphatase in gingival crevicularfluid: Their relations to periodontal status. J Clin Periodontol. 1994; 21:327-33.

5. Binder TA, Goodson JM. Gingival fluid levels of acid and alkaline phosphatase. J Periodontal Res. 1987; 22:14-19.

6. Chapple HC, Matthews JB, Thorpe GH, Glenwright HD, Smith JM, Saxby MS. A new ultrasensitive chemiluminescent assay for the site specific quantification of alkaline phosphatase in gingival crevicular fluid. J Periodontal Res. 1993; 28:266-73.

7. Delmas PD. What do we know about biochemical bone markers? Baillieres Clin Obstet Gynaecol. 1991; 5:817-30.

8. Schreiber W, Whitta L. Alkaline phosphatase isoenzymes resolved by electrophoresis on lectin-containing agarose gel. Clin Chem. 1986; 32:1570-3.

9. Hoof VVO, Lepoutre LG, Hoylaerts MF, Chevign R, De Broe ME. Improved agarose electrophoretic method for separating alkaline phosphatase isoenzymes in serum. Clin Chem. 1998; 34:1857-62.

10. Moss DW, Whitby LG. A simplified heat-inactivation method for investigating alkaline phosphatase isoenzymes in serum. Clin Chim Acta. 1975; 61:63-71.

11. Rosalki SB, Foo AY. Two new methods for separating and quantifying bone and liver alkaline phosphatase isoenzymes in plasma. Clin Chem. 1986; 32:1960-6.

12. Ohlsson C, Isgaard J, Tönell T, Nilsson A, Isaksson OGP, Lindahl A. (1993): Endocrine regulation of longitudinal bone growth [Review]. ActaPaediatr Scand. 82 [Suppl 391]:33

13. Requirand P, Gibert P, Tramini P, Cristol JP, Descomps B. Serum fatty acid imbalance in bone loss: example with periodontal disease. Clin Nutr. 2000; 19:271-6.

14. Gibert P, Tramini P, Sieso V, Piva MT. Alkaline phosphatase isozyme activity in serum from patients with chronic periodontitis. J Periodontal Res. 2003; 38:362-5. 